\title{
Invasive lobular carcinoma: to grade or not to grade
}

\author{
Anita L Bane ${ }^{1}$, Susanna Tjan ${ }^{1}$, Robert K Parkes ${ }^{2}$, Irene Andrulis ${ }^{1,2}$ and Frances P O’Malley ${ }^{1}$ \\ ${ }^{1}$ Department of Pathology and Laboratory Medicine and ${ }^{2}$ Samuel Lunenfeld Research Institute, Mount Sinai \\ Hospital, University of Toronto, Toronto, Canada
}

\begin{abstract}
Grading of invasive ductal carcinoma of no special type using the Nottingham combined histologic grading system provides independent prognostic information. The prognostic utility of grading invasive lobular carcinomas, however, has not been fully elucidated. In addition, the relationship between grade in invasive lobular carcinomas and expression of predictive biomarkers is less certain. The purpose of this study was to correlate histologic grade in invasive lobular carcinoma with known prognostic and predictive markers. All primary resections for invasive mammary carcinomas diagnosed in Mount Sinai Hospital, Toronto, between the years 1996 and 2002 were reviewed $(n=1053)$. Of these cases, 50 were pure invasive lobular carcinoma (incidence 4.7\%). The median age at diagnosis was 64 years. These tumors were graded using the Nottingham combined histologic grading system and analyzed for estrogen receptor, progesterone receptor, HER2/neu and E-cadherin expression. Tumor grade was correlated with tumor size $(P=0.03)$, and the American Joint Committee on Cancer nodal status $(P=0.05)$. Assessment of the individual components of grade showed that the mitotic score was highly correlated with tumor size $(P=0.02)$, lymph node positivity $(P=0.02)$ and overall American Joint Committee on Cancer stage $(P=0.01)$. Estrogen receptor and progesterone receptor were highly expressed irrespective of the grade of tumor. HER2/neu protein overexpression and E-cadherin protein expression was absent in all invasive lobular carcinomas studied. We conclude that pure invasive lobular carcinoma is uncommon and occurs predominantly in postmenopausal women. Increasing tumor grade is correlated with median tumor size and the American Joint Committee on Cancer nodal stage, but not correlated with the expression of estrogen receptor, progesterone receptor, E-cadherin or HER2/neu protein overexpression.

Modern Pathology (2005) 18, 621-628, advance online publication, 17 December 2004; doi:10.1038/modpathol.3800273
\end{abstract}

Keywords: invasive lobular carcinoma; grade; prognostic markers

Invasive mammary carcinomas are broadly classified into no special type (ductal) and special type cancers. ${ }^{1}$ Invasive lobular carcinoma is a member of the latter category representing $0.6-20 \%$ of all invasive cancers. ${ }^{2-4}$

Invasive ductal carcinomas of no special type constitutes the majority of invasive mammary cancers, and they are generally graded using the Nottingham combined histologic grading system, which is a modification of the Bloom and Richardson method. ${ }^{5}$ Histologic grade together with tumor size (measured pathologically) and histologic lymph node stage have been found to have a significant correlation with prognosis. ${ }^{6}$ In addition, the expression of prognostic markers in breast cancer, namely

Correspondence: Dr FP O’Malley, MD, Deptartment of Pathology and Laboratory Medicine, Mount Sinai Hospital, 600 University Avenue, Toronto, Canada M5G 1X5.

E-mail: fomalley@mtsinai.on.ca

Received 10 June 2004; revised 4 August 2004; accepted 5 August 2004; published online 17 December 2004 estrogen receptor and progesterone receptor is grade dependent, as is the overexpression and amplification of HER2/neu. The application of the Nottingham combined histologic grading system to invasive lobular carcinoma is not routine in histopathology reporting. In addition, the relationship of biomarkers to grade in invasive lobular carcinoma is unknown. The objective of this study was to correlate histologic grade in invasive lobular carcinoma with tumor size, lymph node status and biomarker profile.

\section{Materials and methods}

A retrospective review of all primary resections for invasive mammary carcinomas conducted at Mount Sinai Hospital, Toronto, between the January 1996 and December 2002 was undertaken. A total of 1053 invasive mammary carcinomas were diagnosed in this period, 50 of which were pure invasive lobular carcinomas. Strict morphologic criteria were used to 
identify the cohort of invasive lobular carcinomas, namely a monotonous population of discohesive cells devoid of any tubule formation. Evidence of tubule formation in the representative sections of tumor archived made the tumor ineligible for the study and these tumors were classified as invasive ductal, no special type with lobular features.

Hematoxylin and eosin-stained sections of the formalin-fixed, paraffin-embedded tumors were reviewed. The 50 invasive lobular carcinomas were independently graded by two of the authors using the Nottingham combined histologic grading system. ${ }^{6}$ In accordance with this grading system, each tumor was assessed and scored numerically for the percentage of tubule formation, the degree of nuclear pleomorphism and the mitotic count in 10 high-power fields (field diameter $0.44 \mathrm{~mm}$ ). The scores obtained for each morphological parameter were added to obtain an overall grade. In cases where assessment of grade differed, the disagreement was resolved by consensus after joint review using a multiheaded microscope. Tumor size, tumor grade, lymph node status and other prognostic parameters were recorded and tabulated (Table 1).

Representative sections of invasive tumor were selected. Sections of paraffin-embedded tumor, $4 \mu \mathrm{m}$ thick, were cut and stained for estrogen receptor (6F11, Novocastra, dilution 1/70), progesterone receptor (PgR, DAKO, dilution 1/1500), HER2/neu protein (CB11, Novacastra, dilution 1/200 \& A0485, DAKO, dilution 1/750), and E-cadherin (HECD-1, Zymed, prediluted). Estrogen receptor and progesterone receptor were interpreted as positive if $>1 \%$ of tumor cells demonstrated nuclear positivity. ${ }^{7}$ HER2/neu protein overexpression was recorded if $>10 \%$ of tumor cells demonstrated complete, moderate to strong membrane staining. Complete, circumferential membrane staining with the Ecadherin antibody in any percentage of tumor cells was considered positive.

Differences between tumor grades were tested using the Jonckheere-Terpstra nonparametric test for both continuous (age, size, number of positive lymph nodes), binary (positivity of lymph nodes, estrogen receptor, progesterone receptor, Her2/ neu, E-Cadherin) and categorical (American Joint
Committee on Cancer T and N, stage) variables. Categorical variables were treated as ordinal, using the natural ordering of these scoring systems. Exact (not asymptotic) $P$-values were calculated using the SAS software (version 8.2, SAS Institute, Cary NC, USA).

\section{Results}

Of 1053 primary resections for invasive mammary carcinoma conducted at Mount Sinai Hospital, during the study period, 50 cases of pure invasive lobular carcinoma were identified using the strict morphololgical criteria outlined (incidence 4.7\%). Of the tumors, $10(20 \%)$ were grade I (Figure 1), 33 $(66 \%)$ were grade II (Figure 2) and seven (14\%) were grade III (Figure 3), using the Nottingham combined histologic grading system. ${ }^{6}$

The median patient age at the time of diagnosis was 64 (range 37-85) years, and did not differ between tumor grades $(P=0.63)$. The median tumor size, as determined by pathological examination, increased incrementally with increasing tumor grade. The median size was $1.2 \mathrm{~cm}$ for grade I tumors, $2.5 \mathrm{~cm}$ for grade II tumors and $4.4 \mathrm{~cm}$ for grade III tumors $(P=0.03)$. The American Joint

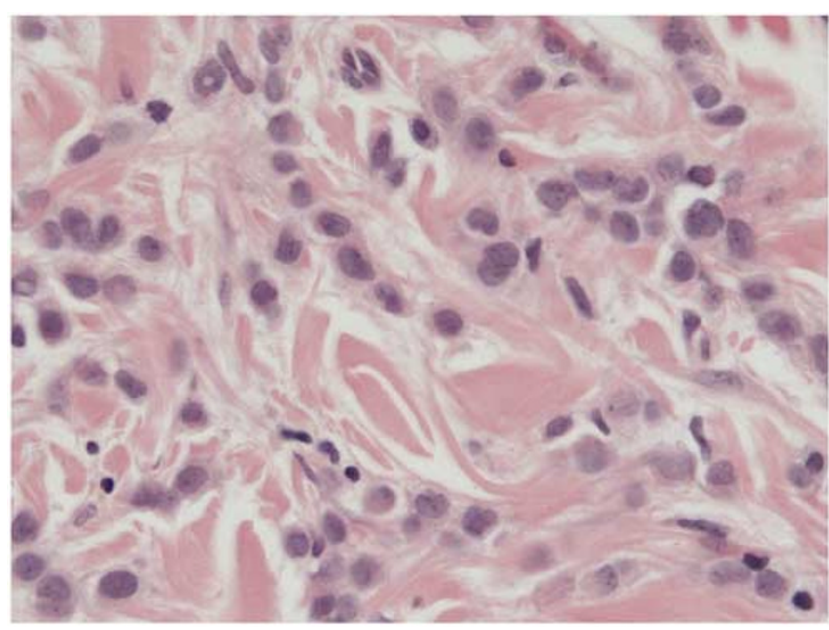

Figure 1 Invasive lobular carcinoma, grade I/III, with cells exhibiting minimal nuclear pleomorphism ( $\times 60$ magnification).

Table 1 Relationship of tumor grade to tumor size, lymph node status, and overall AJCC stage

\begin{tabular}{lcccc}
\hline ILC $(\mathrm{n}=50)$ & Grade I & Grade II & Grade III & Statistical (P) \\
\hline Number & $10(20 \%)$ & $33(66 \%)$ & $7(14 \%)$ & 64 \\
Age (median) (years) & 61 & 66 & 4.4 & 0.63 \\
Median size (cm) & 1.2 & 2.5 & T2 & 83 \\
AJCC T (median) & T1c & T2 & 32 & N1a \\
LN positivity (\%) & 29 & No & IIIA & 0.13 \\
AJCC N (median) & No & IIA & & 0.09 \\
Stage (median) & I & & 0.09
\end{tabular}

ILC: invasive lobular carcinoma; AJCC T: American Joint Committee on Cancer Tumor Stage; LN: lymph node; AJCC N: American Joint Committee on Cancer Nodal Stage. 
Committee on Cancer nodal stage was correlated with tumor grade $(P=0.05)$ with a trend toward increasing tumor grade and both nodal metastases

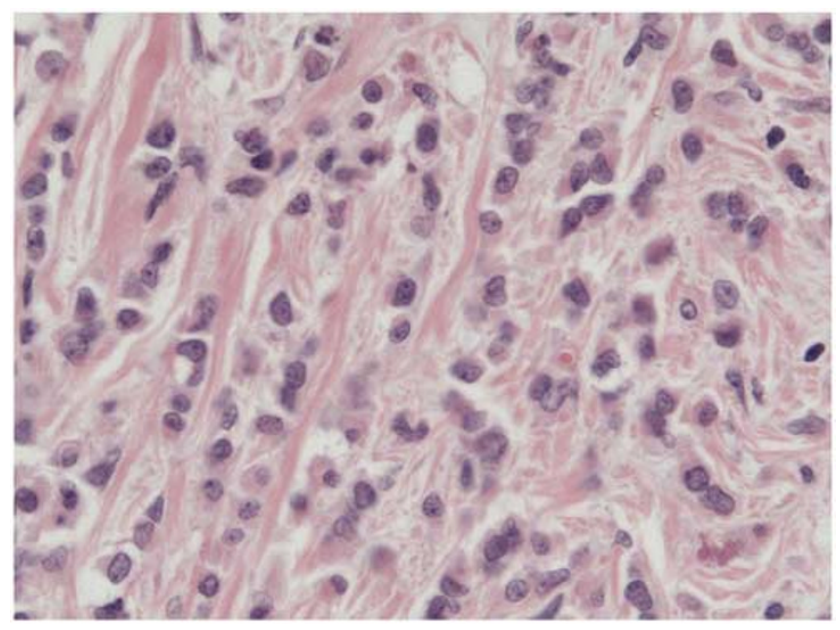

Figure 2 Invasive lobular carcinoma, grade II/III, with cells exhibiting moderate nuclear pleomorphism but maintaining a single file growth pattern $(\times 60$ magnification).

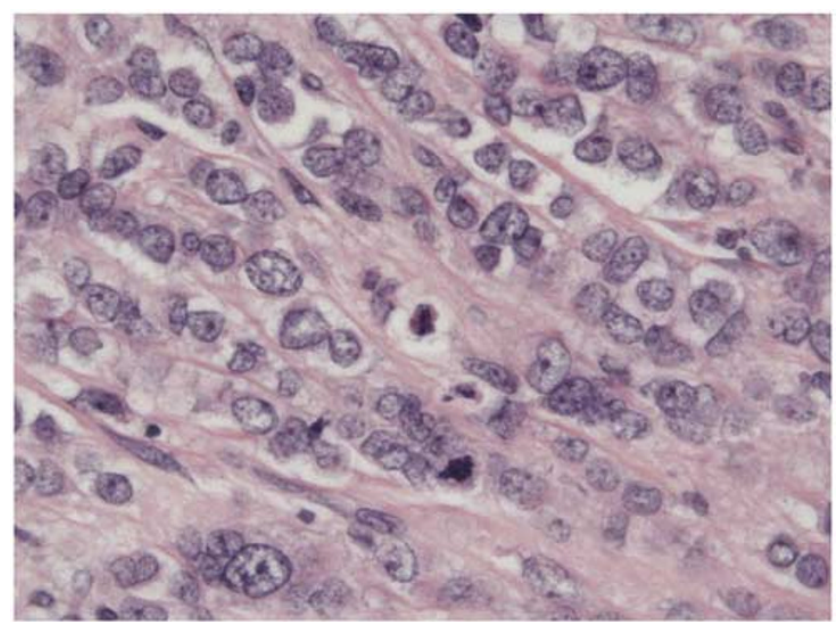

Figure 3 Invasive lobular carcinoma, grade III/III, with cells exhibiting significant nuclear pleomorphism ( $\times 60$ magnification).
$(P=0.08)$ and increasing median number of nodes involved $(P=0.06)$. The overall stage of the tumors at diagnosis (a function of both tumor size and number of lymph nodes involved) increased progressively with tumor grade. The median stage of grade I tumors was stage I, for grade II tumors stage IIA and for grade III tumors stage IIIA $(P=0.08)$. When tumor grade was divided into its constituent parts and re-examined, increasing nuclear grade was associated with increasing tumor size, lymph node positivity and tumor size. None of these parameters reached statistical significance (Table 2). Mitotic count, however, when analyzed in isolation was strongly associated with tumor size $(P=0.02)$, lymph node positivity $(P=0.02)$, and overall American Joint Committee on Cancer stage $(P=0.01)$ (Table 3).

In our cohort, $100 \%$ of tumors expressed estrogen receptor, and progesterone receptor was expressed with high frequency $(>85 \%)$. Both were independent of tumor grade $(P \geq 0.95)$. None of the tumors were found to overexpress HER2/ neu protein. All of the tumors had HER2/neu protein expression assessed by two antibodies and in no case was there an equivocal result necessitating further investigation for gene amplification status using fluorescent in situ hybridization (FISH). E-cadherin was uniformly absent in all invasive lobular carcinomas studied (Table 4).

\section{Discussion}

Lobular carcinoma was first described by Foote and Stewart ${ }^{8,9}$ in 1941. They describe both the in situ form of the disease, confined to the lobule and terminal ducts and the invasive form. The morphologic features that distinguish this neoplasm from ductal carcinoma are two-fold.

I. The cells are monotonous and discohesive with prominent intracytoplasmic lumina.

II. The pattern of invasion is frequently in a single file arrangement with a targatoid growth pattern around terminal ducts.

Table 2 Tumor characteristics by nuclear grade $(n=50)$

\begin{tabular}{|c|c|c|c|c|}
\hline$I L C(\mathrm{n}=50)$ & Nuclear grade I & Nuclear grade II & Nuclear grade III & Significance test \\
\hline Number & 10 & 34 & 6 & \\
\hline Age (median) (years) & 61 & 65 & 64.5 & 0.99 \\
\hline Size (median) (cm) & 1.15 & 2.55 & 2.60 & 0.12 \\
\hline AJCC T (median) & T1c & $\mathrm{T} 2$ & $\mathrm{~T} 2$ & 0.31 \\
\hline LN positivity (\%) & 29 & 38 & 60 & 0.37 \\
\hline Stage (median) & I & IIA & IIA & 0.28 \\
\hline Number of positive lymph nodes (median) & 0 & 0 & 3 & 0.35 \\
\hline AJCC N (median) & No & No & No & 0.25 \\
\hline
\end{tabular}

ILC: invasive lobular carcinoma; AJCC T: American Joint Committee on Cancer Tumor Stage; LN: lymph node; AJCC N: American Joint Committee on Cancer Nodal Stage. 
Table 3 Tumor characteristics by mitotic count per 10 high-power fields, field diameter $0.44 \mathrm{~mm}$

\begin{tabular}{|c|c|c|c|c|}
\hline$I L C(\mathrm{n}=50)$ & $M C 0-5$ & MC 6-10 & $M C>11$ & Significance test \\
\hline Number & 40 & 7 & 3 & \\
\hline Age (median) (years) & 65 & 64 & 48 & 0.22 \\
\hline Size (median) (cm) & 1.7 & 3.0 & 5.0 & 0.02 \\
\hline AJCC T (median) & T1c & $\mathrm{T} 2$ & T3 & 0.03 \\
\hline LN positivity (\%) & 30 & 60 & 100 & 0.02 \\
\hline Stage (median) & IIA & IIB & IIIC & 0.01 \\
\hline Number of positive lymph nodes (median) & 0 & 3 & 11 & 0.007 \\
\hline AJCC N (median) & No & No & N3 & 0.01 \\
\hline
\end{tabular}

ILC: invasive lobular carcinoma; MC: mitotic count; AJCC T: American Joint Committee on Cancer Tumor Stage; LN: lymph node; AJCC N: American Joint Committee on Cancer Nodal Stage.

Table 4 Relationship of tumor grade with the expression of estrogen receptor, progesterone receptor, HER2/neu protein and E-cadherin protein

\begin{tabular}{lcccc}
\hline$I L C(\mathrm{n}=50)$ & Grade I & Grade II & Grade III & Statistical (P) \\
\hline Number & $10(20 \%)$ & $33(66 \%)$ & $7(14 \%)$ & 100 \\
ER (\%) & 100 & 100 & 86 & 1.00 \\
PR (\%) & 90 & 85 & 0 & 0.95 \\
HER2/neu (\%) & 0 & 0 & 0 & 1.00 \\
E-Cadherin (\%) & 0 & 0 & \\
\hline
\end{tabular}

ILC: invasive lobular carcinoma; ER: estrogen receptor; PR: progesterone receptor.

The reported incidence of invasive lobular carcinoma ranges from 0.6 to $20 \% .^{2-4}$ In the present series, the proportion of invasive lobular carcinoma was $4.7 \%$. The variation in reported incidence is most probably due to different histopathological criteria used to define invasive lobular carcinoma rather than a real variation in incidence. We defined invasive lobular carcinoma on the basis of typical cytomorphologic characteristics, cited above and absent ductal formation.

Since the original description of invasive lobular carcinoma, variants other than the 'classical type' have been described. These include, the solid variant, ${ }^{10}$ the alveolar variant, ${ }^{3}$ the tubulo-lobular variant, ${ }^{11}$ the signet ring variant, ${ }^{12}$ the histiocytoid variant $^{13}$ and a mixed subgroup. ${ }^{12}$ The mixed subgroup, in Dixon et al's ${ }^{12}$ original paper, was recognized when an invasive lobular carcinoma was comprised of a mixture of variants or when the invasive lobular carcinoma had a classic growth pattern, but the degree of nuclear pleomorphism was greater than that seen in the classic subgroup. Eusebi et $a l^{14}$ described the clinical outcome in a group of cases he termed 'pleomorphic lobular carcinoma' which had the growth pattern of classic invasive lobular carcinoma but showed at least a moderate degree of nuclear pleomorphism. These tumors were associated with an aggressive behavior with six patients $(60 \%)$ dying of disease within 42 months of diagnosis. Weidner and Semple ${ }^{15}$ divided invasive lobular carcinomas into 'pleomorphic' or 'classical' subtypes, based on nuclear characteristics, similar to the criteria described by Eusebi.
Their study demonstrated a trend toward a decrease in overall survival in patients with pleomorphic invasive lobular carcinoma compared with patients with classic invasive lobular carcinoma. Pleomorphic lobular carcinoma has subsequently been the subject of a number of papers ${ }^{16-19}$ and is described under the general rubric of invasive lobular carcinoma in the new World Health Organisation Classification of Tumours. ${ }^{4}$

Nuclear pleomorphism is only one of the features of the Nottingham combined grading system. ${ }^{6}$ This grading scheme involves semiquantitative evaluation of three morphological features-the percentage of tubule formation, the degree of nuclear pleomorphism and an accurate mitotic count using a defined field area. A numerical scoring system and the overall grade are derived from a summation of individual scores for the three variables: three grades of differentiation are used. ${ }^{6}$ Histologic grade is strongly correlated with prognosis, and is correlated with both disease-free and overall survival. ${ }^{20-23}$ The authors of the original study stressed that this grading system had clinical utility for both invasive lobular carcinoma and special type cancers. This was further validated by the work of Sastre-Garau et al, ${ }^{24}$ who showed that histologic grade is as important for prognosis in invasive lobular carcinoma as it is in invasive ductal carcinomas. However, pathologists have been reluctant to utilize it in relation to invasive lobular carcinoma due primarily, it is thought, to the absence of any tubule formation in invasive lobular carcinomas, rendering that dimension of the three-tiered grading system redundant. 
To investigate grading in invasive lobular carcinoma, du Toit et $a l^{25}$ graded 171 such tumors in accordance with the Nottingham combined histologic grading system, and demonstrated that $20 \%$ were grade I, $64 \%$ grade II and $16 \%$ were grade III or high grade. These figures are similar to those in the current study.

In our cohort of pure invasive lobular carcinomas, we demonstrated that there was a positive correlation between increasing tumor grade and tumor size. The median size of tumors increased with tumor grade (Table 1). This result was statistically significant $(P=0.03)$. Tumor size is a known independent prognostic factor, ${ }^{26-28}$ and the incidence of nodal metastases is known to increase with primary tumor size. ${ }^{28}$

Axillary lymph node status is the single best predictor of patient survival. ${ }^{29}$ The absolute number of involved axillary nodes at the time of diagnosis provides additional prognostic information, which is reflected in the revised American Joint Committee on Cancer nodal staging system. ${ }^{30}$ The number of involved nodes also influences the choice of adjuvant therapy protocols. ${ }^{31}$ In our study of 50 invasive lobular carcinomas, there was a trend toward increasing lymph node involvement and increasing tumor grade $(P=0.08) ; 29 \%$ of grade I tumors, $32 \%$ of grade II tumors and $83 \%$ of grade III tumors had axillary lymph node metastases at diagnosis. The median number of positive nodes was 0 for both grade I and grade II tumors, but patients with grade III tumors had a median number of 3.5 lymph nodes positive at diagnosis $(P=0.06)$. There was a positive correlation between the median nodal stage, as defined by the new American Joint Committee on Cancer staging system and tumor grade. The median ' $\mathrm{N}$ ' stage for grade I and II invasive lobular carcinomas was No but for grade III tumors it was 'N1a'. This result was statistically significant $(P=0.05)$.

The Nottingham combined histologic grading system $^{6}$ assesses three tumor parameters as discussed above. When we analyzed these parameters separately, tubule formation was noninformative as all invasive lobular carcinomas by definition score $3 / 3$. Nuclear pleomorphism, despite being the initial morphologic factor that defined different grades of invasive lobular carcinoma, when analyzed alone showed only a weak correlation with tumor size, lymph node status or overall American Joint Committee on Cancer staging. However, the mitotic score when examined in isolation was highly correlated with all the prognostic parameters assessed (Table 3). These results were highly statistically significant. This fact suggests that the single most important component of the grading system in determining stage and biological aggressiveness in invasive lobular carcinomas is the mitotic index. This is not an unexpected finding as other investigators have assessed mitotic activity in invasive mammary carcinomas not classified by morphologic subtypes and have also shown that it is the strongest predictor of decreased survival and it contributes predictive information beyond that obtained with the combined histologic grade. ${ }^{32-36}$ Indeed multivariate analysis of the Bloom Richardson grading system, ${ }^{5}$ of which the Nottingham combined histologic grading system is a modification, indicates that the mitotic index contributed much of the progno stic value. ${ }^{33}$ Determination of estrogen receptor positivity is now routinely performed by immunohistochemsitry on paraffin-embedded tumor sections. Estrogen receptor status carries both prognostic and predictive information. Estrogen receptor-positive tumors are known to have a better prognosis than estrogen receptor-negative tumors and estrogen receptor status is strongly correlated with tumor grade and differentiation, patient age and tumor size. In the clinical setting, the major importance of estrogen receptor lies in its predictive value. The response of both advanced and early breast cancer to endocrine therapy is proportional to estrogen receptor content. ${ }^{37,38}$ Our study illustrates that all invasive lobular carcinomas irrespective of patient age, tumor size or grade are positive for estrogen receptor and thus carry significant predictive value for the patient. However this data suggests that estrogen receptor status carries no discriminatory prognostic value in the setting of invasive lobular carcinoma. When Frolik et al ${ }^{19}$ graded their invasive lobular carcinomas, $93 \%$ of grade I and grade II and $100 \%$ of grade III invasive lobular carcinomas expressed estrogen receptor. Yet other studies that have addressed estrogen receptor status in pleomorphic lobular carcinomas not otherwise graded, have found varying degrees of receptor positivity, $9-81 \% .^{16-18}$

The expression of progesterone receptor also carries both prognostic and predictive information. The prognostic utility of progesterone receptor has been validated in a number of studies ${ }^{39-45}$ and is thought to reflect the presence of a functional estrogen receptor pathway in the tumor and is used to predict the response of patients to adjuvant endocrine therapy. ${ }^{41}$ In our study cohort, there was a high incidence of progesterone receptor positivity $(85-90 \%)$ that was not correlated with tumor grade $(P=0.95)$. The literature shows that classic invasive lobular carcinomas, which correlate with grade I invasive lobular carcinomas are known to have a high incidence ( $>90 \%$ ) of progesterone receptor expression. ${ }^{16-19}$ The reported incidence, however, of progesterone receptor positivity for 'pleomorphic' lobular carcinomas, corresponding to grade II and III in our study, is widely disparate, ranging from 9 to $97 \% .{ }^{16,18,19}$

HER2/neu is a proto-oncogene located on the long arm of chromosome $17 .^{45}$ Low levels of the protein expressed by this gene are normally present in many adult tissues including breast, endometrium, prostate and ovary. Amplification of this gene and increased levels of the protein product have been found in between 10 and $35 \%$ of invasive breast 
cancers. ${ }^{46}$ The presence of amplification and protein overexpression is associated with a number of adverse clinical outcomes, including decreased overall and disease-free survival, especially for patients with lymph node-positive disease. ${ }^{47-49}$ The presence of amplification carries predictive information with regard to responsiveness to anthracycline-based chemotherapy and is an absolute criterion for treatment with trastuzumab (Herceptin) therapy in the metastatic setting. HER2/neu gene amplification in invasive ductal carcinoma is correlated with grade. Tsuda et $a l^{50}$ found $H E R 2 / n e u$ amplification in $33 \%$ of grade III invasive ductal carcinomas, $10 \%$ of grade II carcinomas and $0 \%$ of grade I carcinomas. Similar results were obtained in our own institution. ${ }^{51}$ The amplification of this gene has been found in $0 \%$ of special type cancers and $<1 \%$ of invasive lobular carcinomas. ${ }^{52}$ In the latter study, only one of 67 invasive lobular carcinomas was positive for HER2/neu gene amplification. This was designated as a 'pleomorphic' subtype of invasive lobular carcinoma with a nuclear grade of III/III. No reference to overall Nottingham combined histologic grade was given. In studies assessing HER2/neu overexpression by immunohistochemistry the results are highly variable. Middleton et $a l^{17}$ found HER2/neu overexpression in $81 \%$ of 'pleomorphic' invasive lobular carcinomas not further stratified by grade. Frolik et $a l^{19}$ found overexpression in $53 \%$ of grade III invasive lobular carcinomas and $6.66 \%$ of both grades II and I invasive lobular carcinomas, whereas none of the 15 cases of invasive lobular carcinomas studied by Porter et al ${ }^{53}$ showed HER2/neu overexpression. Of the 50 invasive lobular carcinomas studied in our institution, none showed HER2/neu protein overexpression as assessed by immunohistochemical staining with two different antibodies. The lack of HER2/neu overexpression may be reflective of the small number of cases in this study. Indeed, in our consultation service, we have a small number of grade III invasive lobular carcinomas that are positive for HER2/neu protein overexpression and gene amplification (unpublished data). The discrepancy in the reported rates of HER2/neu overexpression in invasive lobular carcinoma may be accounted for by variation in histological classification or antibody sensitivity and specificity. Our results suggest that $H E R 2 / n e u$ gene amplification is of minor importance in the development or progression of invasive lobular carcinoma regardless of grade, in contrast with invasive ductal carcinomas. Molecular characterization of invasive lobular carcinomas has shown that they have a higher incidence of loss of chromosome $17 q$ than ductal carcinomas $(P=0.3)$ and that ductal tumors have a higher incidence of 17q22-24 amplification than their lobular counterparts, ${ }^{54}$ factors that may influence HER2/neu gene copy number and hence protein expression. A cDNA microarray study similarly showed that only two of 17 lobular carcinomas arrayed displayed HER2/neu gene overexpression. ${ }^{55}$
E-cadherin is a calcium-dependent cell-cell adhesion molecule that is localized in lateral cell-cell contacts and enriched in the zonula adherens junction. Its function is to mediate intercellular adhesion via homophillic interactions. ${ }^{56}$ It plays an essential role in the formation and maintenance of normal architecture and function of epithelial tissues. ${ }^{57}$ It also shows an important invasion suppression activity. ${ }^{58}$ The gene encoding the human E-cadherin, $C D H 1$ is localized on chromosome 16q22.1. Chromosome $16 q$ is frequently lost in invasive lobular carcinomas and somatic mutations in this gene have been found in over $50 \%$ of invasive lobular carcinomas. ${ }^{58}$ Somatic mutations are frequently accompanied by loss of the normal allele by loss of heterozygosity or alternatively by epigenetic silencing, signature features of a tumor suppressor gene. Loss of heterozygosity at the E-cadherin locus has been demonstrated in 27 of 27 invasive 'pleomorphic' lobular carcinomas in one study. ${ }^{59}$ In most cases, the mutations are of the truncating type resulting in loss of protein expression, ${ }^{60}$ which is compatible with the typical dispersed tumor growth with obvious loss of cell-cell adhesion. Lack of the protein expression can be assessed using immunohistochemistry. E-cadherin expression is normally present in the epithelial cell membranes in accordance with its function with cell adhesion. In our series, all tumors were negative for Ecadherin expression irrespective of grade, suggesting that loss of E-cadherin is a function of tumor morphology and not biological aggressiveness and is of no prognostic importance. Other authors have found E-cadherin expression in $0-45 \%$ of invasive lobular carcinomas. ${ }^{59-68}$ In these studies no correlation with grade was made. The discrepancy in the literature with regard to the percentage of invasive lobular carcinomas expressing E-cadherin may relate to differences between the populations studied, sensitivity and specificity of the antibodies used and diagnostic variation as applied to lobular carcinomas.

We have shown that invasive lobular carcinoma when characterized by strict morphological criteria is uncommon. Attempts have previously been made to subdivide invasive lobular carcinomas on the basis of morphological parameters and more recently by assessing 'nuclear pleomorphism'. We have applied the well-standardized and widely accepted Nottingham combined histologic grading system to a sequential series of invasive lobular carcinomas from one institution. We have correlated our results with tumor factors of known prognostic and predictive importance. We have shown that grade is correlated with overall tumor size and the American Joint Committee on Cancer nodal status. In contrast, estrogen receptor and progesterone receptor expression and HER2/neu overexpression are not correlated with grade. Expression of the tumor suppressor gene/cell adhesion factor, Ecadherin is universally lost in all invasive lobular 
carcinomas irrespective of grade suggesting that its loss is a pre-requisite for the lobular phenotype but it does not dictate biological aggressiveness. It is clear from this study that invasive lobular carcinoma is both morphologically and biologically distinct from invasive ductal carcinomas and that grading is important. Further studies correlating the grade of invasive lobular carcinoma and response to adjuvant treatments and long-term survival are necessary to expand the body of knowledge concerning invasive lobular carcinomas.

\section{References}

1 Page DL. Special types of invasive breast cancer, with clinical implications. Am J Surg Pathol 2003;27: 832-835.

2 Correa P, Johnson W. International variation in the histology of breast carcinoma. UICC Tech Rep Ser 1978;35:36-65.

3 Martinez V, Azzopardi JG. Invasive lobular carcinoma of the breast: incidence and variants. Histopathology 1979;3:467-488.

4 Tavassoli FA, Devilee P. Pathology and Genetics of Tumours of the Breast and Female Genital Organs. World Health Organisation of Classification of Tumours. IARC Press: Lyon, 2003.

5 Bloom H, Richardson W. Histological grading and prognosis in breast cancer. Br J Cancer 1957;11:359-377.

6 Elston CW, Ellis IO. Pathological prognostic factors in breast cancer. I. The value of histological grade in breast cancer: experience from a large study with longterm follow-up. Histopathology 1991;19:403-410.

7 Harvey JM, Clark GM, Osborne CK, et al. Estrogen receptor status by immunohistochemistry is superior to the ligand-binding assay for predicting response to adjuvant endocrine therapy in breast cancer. J Clin Oncol 1999;17:1474-1481.

8 Foote F, Stewart F. Lobular carcinoma in situ. Am J Pathol 1941;17:491-496.

9 Foote F, Stewart F. A histological classification of carcinoma of the breast. Surgery 1946;19:74-99.

10 Fechner RE. Histologic variants of infiltrating lobular carcinoma of the breast. Hum Pathol 1975;6:373-378.

11 Fisher ER, Gregorio RM, Redmond C, et al. Tubulolobular invasive breast cancer: a variant of lobular invasive cancer. Hum Pathol 1977;8:679-683.

12 Dixon JM, Anderson TJ, Page DL, et al. Infiltrating lobular carcinoma of the breast. Histopathology 1982; 6:149-161.

13 Walford N, ten Velden J. Histiocytoid breast carcinoma: an apocrine variant of lobular carcinoma. Histopathology 1989;14:515-522.

14 Eusebi V, Magalhaes F, Azzopardi JG. Pleomorphic lobular carcinoma of the breast: an aggressive tumor showing apocrine differentiation. Hum Pathol 1992; 23:655-662.

15 Weidner N, Semple JP. Pleomorphic variant of invasive lobular carcinoma of the breast. Hum Pathol 1992; 23:1167-1171.

16 Bentz JS, Yassa N, Clayton F. Pleomorphic lobular carcinoma of the breast: clinicopathologic features of 12 cases. Mod Pathol 1998;11:814-822.

17 Middleton LP, Palacios DM, Bryant BR, et al. Pleomorphic lobular carcinoma: morphology, immuno- histochemistry, and molecular analysis. Am J Surg Pathol 2000;24:1650-1656.

18 Radhi JM. Immunohistochemical analysis of pleomorphic lobular carcinoma: higher expression of p53 and chromogranin and lower expression of ER and PgR. Histopathology 2000;36:156-160.

19 Frolik D, Caduff R, Varga Z. Pleomorphic lobular carcinoma of the breast: its cell kinetics, expression of oncogenes and tumour suppressor genes compared with invasive ductal carcinomas and classical infiltrating lobular carcinomas. Histopathology 2001; 39:503-513.

20 Davis BW, Gelber RD, Goldhirsch A, et al. Prognostic significance of tumor grade in clinical trials of adjuvant therapy for breast cancer with axillary lymph node metastasis. Cancer 1986;58:2662-2670.

21 Freedman LS, Edwards DN, McConnell EM, et al. Histological grade and other prognostic factors in relation to survival of patients with breast cancer. $\mathrm{Br}$ J Cancer 1979;40:44-55.

22 Page DL. Prognostic indicators in breast cancer and who needs them. Anat Pathol 1997;2:35-52.

23 Pereira H, Pinder SE, Sibbering DM, et al. Pathological prognostic factors in breast cancer. IV: Should you be a typer or a grader? A comparative study of two histological prognostic features in operable breast carcinoma. Histopathology 1995;27:219-226.

24 Sastre-Garau X, Jouve M, Asselain B, et al. Infiltrating lobular carcinoma of the breast. Clinicopathologic analysis of 975 cases with reference to data on conservative therapy and metastatic patterns. Cancer 1996;77:113-120.

$25 \mathrm{du}$ Toit RS, Locker AP, Ellis IO, et al. Invasive lobular carcinomas of the breast-the prognosis of histopathological subtypes. Br J Cancer 1989;60:605-609.

26 Carter CL, Allen C, Henson DE. Relation of tumor size, lymph node status, and survival in 24,740 breast cancer cases. Cancer 1989;63:181-187.

27 Fisher B, Slack NH, Bross ID. Cancer of the breast: size of neoplasm and prognosis. Cancer 1969;24:1071-1080.

28 Say CC, Donegan WL. Invasive carcinoma of the breast: prognostic significance of tumor size and involved axillary lymph nodes. Cancer 1974;34:468-471.

29 Fisher B, Bauer M, Wickerham DL, et al. Relation of number of positive axillary nodes to the prognosis of patients with primary breast cancer. An NSABP update. Cancer 1983;52:1551-1557.

30 Singletary SE, Allred C, Ashley P, et al. Revision of the American Joint Committee on Cancer staging system for breast cancer. J Clin Oncol 2002;20:3628-3636.

31 Harris J. Radiation therapy: accepted and controversial roles. Breast J 2003;9:S11-S16.

32 Baak JP. Mitosis counting in tumors. Hum Pathol 1990;21:683-685.

33 Clayton F. Pathologic correlates of survival in 378 lymph node-negative infiltrating ductal breast carcinomas. Mitotic count is the best single predictor. Cancer 1991;68:1309-1317.

34 Simpson JF, Gray R, Dressler LG, et al. Prognostic value of histologic grade and proliferative activity in axillary node-positive breast cancer: results from the Eastern Cooperative Oncology Group Companion Study, EST 4189. J Clin Oncol 2000;18:2059-2069.

35 van Diest PJ, Baak JP. The morphometric prognostic index is the strongest prognosticator in premenopausal lymph node-negative and lymph node-positive breast cancer patients. Hum Pathol 1991;22:326-330. 
36 Veronese SM, Maisano C, Scibilia J. Comparative prognostic value of Ki-67 and MIB-1 proliferation indices in breast cancer. Anticancer Res 1995;15: 2717-2722.

37 Jordan VC. Third Annual William L. McGuire Memorial Lecture. 'Studies on the estrogen receptor in breast cancer'-20 years as a target for the treatment and prevention of cancer. Breast Cancer Res Treat 1995; 36:267-285.

38 Early Breast Cancer Trialists' Collaborative Group. Tamoxifen for early breast cancer: an overview of the randomised trials. Lancet 1998;351:1451-1467.

39 Bonomi P, Gale M, Von Roenn J, et al. Quantitative estrogen and progesterone receptor levels related to progression-free interval in advanced breast cancer patients treated with megestrol acetate or tamoxifen. Semin Oncol 1988;15:26-33.

40 Encarnacion CA, Ciocca DR, McGuire WL, et al. Measurement of steroid hormone receptors in breast cancer patients on tamoxifen. Breast Cancer Res Treat 1993;26:237-246

41 Ferno M, Baldetorp B, Bendahl PO, et al. Recurrencefree survival in breast cancer improved by adjuvant tamoxifen-especially for progesterone receptor positive tumors with a high proliferation. Breast Cancer Res Treat 1995;36:23-34.

42 Lamy PJ, Pujol P, Thezenas S, et al. Progesterone receptor quantification as a strong prognostic determinant in postmenopausal breast cancer women under tamoxifen therapy. Breast Cancer Res Treat 2002;76: 65-71.

43 Raemaekers JM, Beex LV, Pieters GF, et al. Progesterone receptor activity and relapse-free survival in patients with primary breast cancer: the role of adjuvant chemotherapy. Breast Cancer Res Treat 1987;9: 191-199.

44 Ravdin PM, Green S, Dorr TM, et al. Prognostic significance of progesterone receptor levels in estrogen receptor-positive patients with metastatic breast cancer treated with tamoxifen: results of a prospective Southwest Oncology Group study. J Clin Oncol 1992; 10:1284-1291.

45 Schechter AL, Hung MC, Vaidyanathan L, et al. The neu gene: an erbB-homologous gene distinct from and unlinked to the gene encoding the EGF receptor. Science 1985;229:976-978.

46 Ross JS, Fletcher JA. HER-2/neu (c-erb-B2) gene and protein in breast cancer. Am J Clin Pathol 1999; 112:S53-S67.

47 Paik S, Hazan R, Fisher ER, et al. Pathologic findings from the National Surgical Adjuvant Breast and Bowel Project: prognostic significance of erbB-2 protein overexpression in primary breast cancer. J Clin Oncol 1990;8:103-112.

48 Tandon AK, Clark GM, Chamness GC, et al. HER-2/neu oncogene protein and prognosis in breast cancer. J Clin Oncol 1989;7:1120-1128.

49 Wright C, Angus B, Nicholson S, et al. Expression of c-erbB-2 oncoprotein: a prognostic indicator in human breast cancer. Cancer Res 1989;49:2087-2090.

50 Tsuda H, Hirohashi S, Shimosato Y, et al. Correlation between histologic grade of malignancy and copy number of c-erbB-2 gene in breast carcinoma. A retrospective analysis of 176 cases. Cancer 1990;65: 1794-1800.

51 O’Malley FP, Parkes R, Latta E, et al. Comparison of HER2/neu status assessed by quantitative polymerase chain reaction and immunohistochemistry. Am J Clin Pathol 2001;115:504-511.

52 Hoff ER, Tubbs RR, Myles JL, et al. HER2/neu amplification in breast cancer: stratification by tumor type and grade. Am J Clin Pathol 2002;117:916-921.

53 Porter PL, Garcia R, Moe R, et al. C-erbB-2 oncogene protein in in situ and invasive lobular breast neoplasia. Cancer 1991;68:331-334.

54 Gunther K, Merkelbach-Bruse S, Amo-Takyi BK, et al. Differences in genetic alterations between primary lobular and ductal breast cancers detected by comparative genomic hybridization. J Pathol 2001;193: 40-47.

55 Korkola JE, Devries S, Fridlyand J, et al. Differentiation of lobular versus ductal breast carcinomas by expression microarray analysis. Cancer Res 2003;63: 7167-7175.

56 Takeichi M. Cadherin cell adhesion receptors as a morphogenetic regulator. Science 1991;251:14511455.

57 Takeichi M. Morphogenetic roles of classic cadherins. Curr Opin Cell Biol 1995;7:619-627.

58 Berx G, Becker KF, Hofler H, et al. Mutations of the human E-cadherin (CDH1) gene. Hum Mutat 1998; 12:226-237.

59 Palacios J, Sarrio D, Garcia-Macias MC, et al. Frequent E-cadherin gene inactivation by loss of heterozygosity in pleomorphic lobular carcinoma of the breast. Mod Pathol 2003;16:674-678.

60 Berx G, Cleton-Jansen AM, Strumane K, et al. E-cadherin is inactivated in a majority of invasive human lobular breast cancers by truncation mutations throughout its extracellular domain. Oncogene 1996; 13:1919-1925.

61 Acs G, Lawton TJ, Rebbeck TR, et al. Differential expression of E-cadherin in lobular and ductal neoplasms of the breast and its biologic and diagnostic implications. Am J Clin Pathol 2001;115: 85-98.

62 de Leeuw WJ, Berx G, Vos CB, et al. Simultaneous loss of E-cadherin and catenins in invasive lobular breast cancer and lobular carcinoma in situ. J Pathol 1997; 183:404-411.

63 Droufakou S, Deshmane V, Roylance R, et al. Multiple ways of silencing E-cadherin gene expression in lobular carcinoma of the breast. Int J Cancer 2001; 92:404-408

64 Gamallo C, Palacios J, Suarez A, et al. Correlation of E-cadherin expression with differentiation grade and histological type in breast carcinoma. Am J Pathol 1993;142:987-993.

65 Lehr HA, Folpe A, Yaziji H, et al. Cytokeratin 8 immunostaining pattern and E-cadherin expression distinguish lobular from ductal breast carcinoma. Am J Clin Pathol 2000;114:190-196.

66 Moll R, Mitze M, Frixen UH, et al. Differential loss of E-cadherin expression in infiltrating ductal and lobular breast carcinomas. Am J Pathol 1993;143: 1731-1742.

67 Siitonen SM, Kononen JT, Helin HJ, et al. Reduced E-cadherin expression is associated with invasiveness and unfavorable prognosis in breast cancer. Am J Clin Pathol 1996;105:394-402.

68 Wahed A, Connelly J, Reese T. E-cadherin expression in pleomorphic lobular carcinoma: an aid to differentiation from ductal carcinoma. Ann Diagn Pathol 2002;6:349-351. 